\title{
A Novel Responsivity Model for Stripe-Shaped Ultraviolet Photodiode
}

\author{
Yongjia Zhao', Xiaoya Zhou ${ }^{1}$, Xiangliang Jin $^{1 *}$, Kehan $\mathrm{Zhu}^{2}$ \\ ${ }^{1}$ Faculty of Materials, Optoelectronics and Physics, Xiangtan University, Xiangtan, China \\ ${ }^{2}$ Electrical and Computer Engineering Department, Boise State University, Boise, USA \\ Email: ${ }^{*}$ jinxl@xtu.edu.cn
}

Received June 29, 2012; revised September 12, 2012; accepted September 19, 2012

\begin{abstract}
A novel responsivity model, which is based on the solution of transport and continuity equation of carriers generated both in vertical and lateral PN junctions, is proposed for optical properties of stripe-shaped silicon ultraviolet (UV) photodiodes. With this model, the responsivity of the UV photodiode can be estimated. Fabricated in a standard $0.5 \mu \mathrm{m}$ CMOS process, the measured spectral responsivity of the stripe-shaped UV photodiode shows a good match with the numerical simulation result of the responsivity model at the spectral of UV range. It means that the responsivity model, which is used for stripe-shaped UV photodiode, is reliable.
\end{abstract}

Keywords: Responsivity; Model; Lateral PN Junction; Stripe-Shaped; Silicon; Ultraviolet (UV) Photodiode; CMOS

\section{Introduction}

UV detectors which is invented after infrared and laser detection technology, are widely used in many application areas such as biology, medicine and environmental monitoring [1]; flame detection [2]; space ionizing radiation detection [3], personal radiation protection, semiconductor process control [4]; optical storage system [5]; ultraviolet warning, ultraviolet communication, UV/infrared composite guidance and missile detection.

As UV detectors being developed toward high responsivity and high selectivity, numerical model acts as a design guide to better realization of the performances becomes an essential. UV detectors usually have stripedshaped anode structure; carrier generated in this region can travel vertically and laterally. Although different models for detectors have been presented, most of them only consider carriers transfer vertically, and ignore the transmission in lateral. Dead space effects on gain and noise have been studied in [6-8], but optical characteristic is not considered. UV responsivity models proposed by Pauchard $[9,10]$ showed a good selectivity, but it ignored the influence of lateral PN junction. A two dimensional model proposed in [11] took transverse diffusion into consideration, but longitudinal diffusion is ignored in this model.

In this work, a two dimensional responsivity model for stripe-shaped UV photodiodes is presented. As there is no similar model, silicon test results are used to verify the

${ }^{*}$ Corresponding author. validity of the this work, so simulation results and test results are all needed in this paper. In the next section, the structure and its characteristics are introduced and analyzed. The responsivity model of the stripe-shaped UV photodiode is given in Section 3. The numerical simulation result and silicon test result are presented and discussed in Sections 4 and 5, respectively. Test devices are fabricated in standard $0.5 \mu \mathrm{m}$ CMOS process.

\section{Structure and Characteristics of Stripe-Shaped UV Photodiode}

The device consists of several $\mathrm{P}^{+}$-type anodes, which are implemented by boron implantation in $\mathrm{N}$-well, the $\mathrm{P}^{+}$ anodes are connected by metal outside of the photosensitive region. The substrate is P-type; its electrode is connected with $\mathrm{N}^{+}$electrode and leads to restrained photon absorption in N-well/P-sub junction. The width $\mathrm{W}$ of the stripes is $3.8 \mu \mathrm{m}$. The distance $\mathrm{D}$ between two adjacent stripes is $7.7 \mu \mathrm{m}$. The total sensor area is about $103 \times$ $103 \mu \mathrm{m}^{2}$. The upper $\mathrm{P}^{+} \mathrm{N}$ junctions are at a depth of 140 $\mathrm{nm}$. When the device is biased at its breakdown voltage, the photosensitive region of the photodiode is bounded by the structure surface and the potential barrier $x_{b}\left(x_{b}=\right.$ $800 \mathrm{~nm})$.

When the photodiode is under illumination, UV photons are absorbed at the silicon surface, and these photogenerated carriers are separated by upper $\mathrm{P}^{+} \mathrm{N}$ junction. The photons in visible and infrared range absorbed in deeper junction, but as the N-well-P-substrate junction is short circuited, these photo-generated carriers recom- 
bined and won't contribute to total photocurrent. Thus, the influence of deeper junction can be ignored.

\section{Responsivity Model of the Stripe-Shaped UV Photodiode}

The exposed part of the stripe-shaped photodiode features a periodic distribution, so that it is adequate to investigate the section within the dashed rectangular area as indicated in Figure 1 to present the whole photodiode structure. Presuming the credibility of the depletion-approximation, there can define two quasi-neutral regions I, II and one space charge region A within the dashed rectangular area, as shown in Figure 2. Otherwise, the space between two adjacent anodes is assumed entirely depleted.

Assuming the minority carrier in region I, II is totally generated by diffusion. The contribution of the current in quasi-region to the total photocurrent of the photodiode is elicited by solving the following diffusion equation:

$$
\begin{array}{r}
\frac{d^{2} \Delta n(x, y)}{\mathrm{d} x^{2}}+\frac{d^{2} \Delta n(x, y)}{\mathrm{d} y^{2}}-\frac{\Delta n(x, y)}{L_{n}^{2}}=-\frac{\tau}{L_{n}^{2}} g(x, \lambda) \quad \text { (1) } \quad \begin{array}{l}
\text { carrier distribution, assuming } \Delta n(x, \quad \text { that is to say carrier diffusion current } \\
\text { is independent. Then, Equation (1) }
\end{array} \\
\Delta n(y) \cdot \frac{d^{2} \Delta n(x)}{\mathrm{d} x^{2}}+\Delta n(x) \cdot \frac{d^{2} \Delta n(y)}{\mathrm{d} y^{2}}-\frac{\Delta n(x) \cdot \Delta n(y)}{L_{n}^{2}}=-\frac{\tau}{L_{n}^{2}} g(x, \lambda)
\end{array}
$$

where $\Delta n(x, y)$ indicates the excess minority carrier density, $\tau$ and $L_{n}=\sqrt{D_{n} \tau}$ refers to its lifetime and diffusion length, respectively; see Table $1 g(x, \lambda)$ is photogeneration rate within silicon material, it is given as below:

$$
g(x, \lambda)=P \eta[1-R(\lambda)] a(\lambda) \exp [-a(\lambda) x] /(h v)
$$

where $P$ is the optical power, $\eta$ is the quantum efficiency, $R(\lambda)$ is the surface reflection rate, $\alpha(\lambda)$ the optical absorption coefficient, $h$ and $v$ is the Planck's constant and optical frequency, respectively. The absorption coefficient [12] is related via:

$$
\alpha(\lambda)=\frac{g_{1} h c}{\lambda} \exp \left(\frac{b_{2} h c}{\lambda}\right)
$$

where $g_{1}=1.23 \times 10^{-3} \mu \mathrm{m}^{-1} \cdot \mathrm{ev}^{-1}, b_{2}=2.42 \mathrm{ev}^{-1}$. Figure 3 shows the curve of wavelength vs absorption coefficient.

As the carriers in quasi-neural region-I have two-dimensional distribution. To simplify the complexity of carrier distribution, assuming $\Delta n(x, y)=\Delta n(x) \cdot \Delta n(y)$, that is to say carrier diffusion current in $x$-axis and $y$-axis is independent. Then, Equation (1) can be given

It can be written as

$$
\frac{d^{2} \Delta n(y)}{\mathrm{d} y^{2}}+\frac{\Delta n(y)}{\Delta n(x)}\left(\frac{d^{2} \Delta n(x)}{\mathrm{d} x^{2}}-\frac{\Delta n(x)}{L_{n}^{2}}\right)=-\frac{\tau}{\Delta n(x) L_{n}^{2}} g(x, \lambda)
$$

If only considered electron diffusion current in $x$ direction, i.e. $\Delta n(y)=1$. Then, with boundary conditions $\left(\frac{d}{\mathrm{~d} x} \Delta n\right)_{x=0}=0$ and $\Delta n\left(x_{j}\right)=0$, solving the diffusion equation

$$
\frac{d^{2} \Delta n(x)}{\mathrm{d} x^{2}}-\frac{\Delta n(x)}{L_{n}^{2}}=-\frac{\tau}{L_{n}^{2}} g(x, \lambda)
$$

The $y$-dependency of electron distribution density can be concluded

$$
\Delta n(x)=\frac{\alpha(\lambda) P[1-R(\lambda)] \tau\left[e^{\frac{x_{j}\left[1-\alpha(\lambda) L_{n}\right]-x}{L_{n}}}-e^{-\alpha(\lambda) x}+e^{\frac{x_{j}\left[1-\alpha(\lambda) L_{n}\right]+x}{L_{n}}}-e^{\frac{2 x_{j}}{L_{n}}-\alpha(\lambda) x}+\alpha(\lambda) e^{\frac{2 x_{j}-x}{L_{n}}}-\alpha(\lambda) L_{n} e^{\frac{x}{L_{n}}}\right]}{\left(1+e^{\frac{2 x_{j}}{L_{n}}}\right)\left[-1+\alpha^{2}(\lambda) L_{n}{ }^{2}\right](h v)}
$$

If considering diffusion current in both $x$-axis and $y$-axis. Comparing Equations (5) and (6) leads to

$$
\frac{d^{2} \Delta n(y)}{\mathrm{d} y^{2}}-\frac{\tau g(x, \lambda)}{\Delta n(x) L_{n}^{2}} \Delta n(y)=-\frac{\tau g(x, \lambda)}{\Delta n(x) L_{n}^{2}}
$$

To solving Equation (8), the boundary conditions are assumed to be $\Delta n(x, 0)=0$ and $\Delta n(x, w)=0$, then

$$
\Delta n(y)=\frac{1}{e^{2 \sqrt{H} w}-1} e^{\sqrt{H} y}-\frac{1}{e^{2 \sqrt{H} w}-1} e^{-\sqrt{H} y}+1
$$

where

$$
H=\frac{\tau_{n}}{L_{n}^{2}} \frac{\int_{0}^{x_{j}} P g(x, \lambda) \mathrm{d} x}{\int_{0}^{x_{j}} \Delta n(x) \mathrm{d} x}
$$

As the minority carrier distribution $\Delta n(x, y)=\Delta n(x) \cdot \Delta n(y)$ in region I is known, the components of diffusion current density can be calculated

$$
I_{x_{j}}=\left.q D_{n} \int_{0}^{W} \frac{d \Delta n(x, y)}{\mathrm{d} x}\right|_{x=x_{j}} \mathrm{~d} y
$$




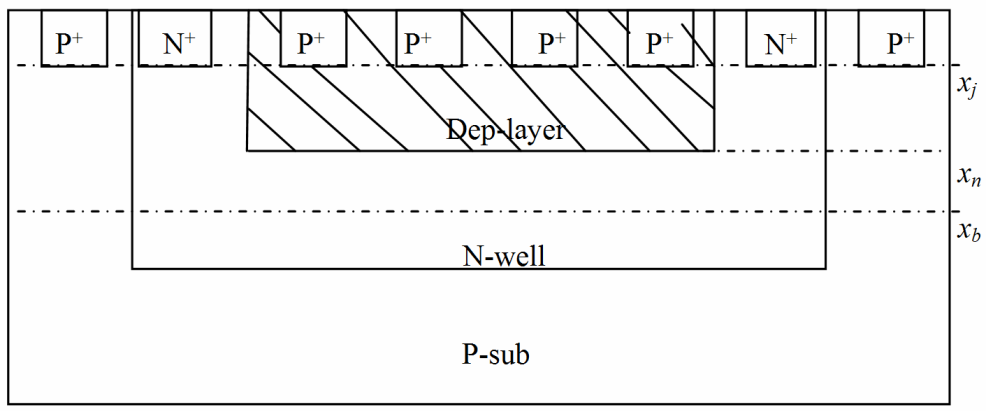

Figure 1. Cross-section view of the stripe-shaped UV photodiode.

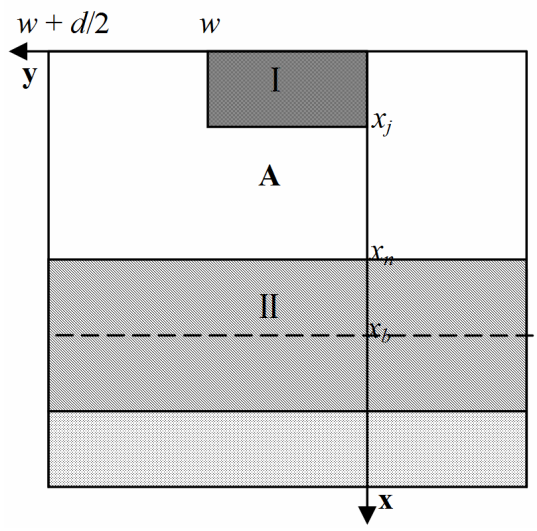

Figure 2. The section within the dashed rectangular area.

Table 1. Some parameters used in this paper.

\begin{tabular}{llll}
\hline Parameter & Name & Parameter & Name \\
\hline$w$ & with of $\mathrm{P}^{+}$anode & $\mathrm{d}$ & distance of two adjcent anode \\
$\Delta n(x, y)$ & the excess minority carrier density & $\tau$ & minority carrier lifetime \\
$L_{n}$ & diffusion length & $\Delta n(x)$ & carrier diffusion in $x$-axis \\
$g(x, \lambda)$ & photo-generation rate within silicon material & $\Delta n(x)$ & carrier diffusion in $y$-axis \\
$P$ & the optical power & $\eta$ & the quantum efficiency \\
$R(\lambda)$ & the surface reflection rate & $\alpha(\lambda)$ & optical absorption coefficient \\
$h$ & the Planck's constant & $v$ & optical frequency \\
\hline
\end{tabular}

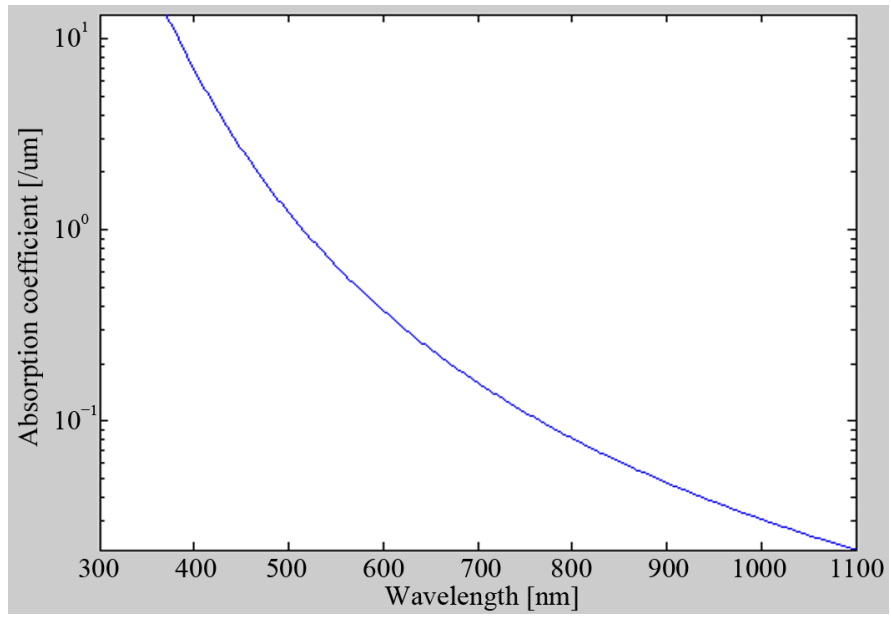

Figure 3. Calculated wavelength vs absorption coefficient based Equation (3) using Matlab. 


$$
\begin{gathered}
I_{I, \text { left }}=\left.q D_{n} \int_{0}^{x_{j}} \frac{d \Delta n(x, y)}{\mathrm{d} y}\right|_{y=w} \mathrm{~d} x \\
I_{I, \text { right }}=\left.q D_{n} \int_{0}^{x_{j}} \frac{d \Delta n(x, y)}{\mathrm{d} y}\right|_{y=0} \mathrm{~d} x
\end{gathered}
$$

The drift current in region $\mathrm{A}$ is obtained by integration of generation rate of electron-hole pairs $g(x, \lambda)$ over the whole depletion region

$$
I_{A}=q d \int_{0}^{x_{n}} g(x, \lambda) \mathrm{d} x+q w \int_{x_{j}}^{x_{n}} g(x, \lambda) \mathrm{d} x
$$

Assuming the distance between $x_{n}$ and $x_{b}$ is much smaller than diffusion length of electrons, which are a few microns. Thus, all carriers generated by photon absorption in region II diffuse toward region A. The electron diffusion current in region II can be expressed using

$$
I_{\Pi}=q(w+d) \int_{x_{n}}^{x_{b}} g(x, \lambda) \mathrm{d} x
$$

Adding all the current generated in region I, II and A, then multiplying the number of fingers, we conclude the total photocurrent of the photodiode

$$
\begin{aligned}
I_{p h}= & 4\left(I_{x_{j}}+I_{I, \text { left }}+I_{I, \text { right }}+I_{A}+I_{\Pi}\right) \\
= & 4\left(q D _ { n } \left(\left.\int_{0}^{w} \frac{d \Delta n(x, y)}{\mathrm{d} x}\right|_{x=x_{j}} \mathrm{~d} y+\left.\int_{0}^{x_{j}} \frac{d \Delta n(x, y)}{\mathrm{d} y}\right|_{y=w} \mathrm{~d} x\right.\right. \\
& \left.+\left.\int_{0}^{x_{j}} \frac{d \Delta n(x, y)}{\mathrm{d} y}\right|_{y=0} \mathrm{~d} x\right)+q d \int_{0}^{x_{n}} g(x, \lambda) \mathrm{d} x \\
& \left.+q w \int_{x_{j}}^{x_{n}} g(x, \lambda) d x+q(w+d) \int_{x_{n}}^{x_{b}} g(x, \lambda) \mathrm{d} x\right)
\end{aligned}
$$

\section{Numerical Simulation Result}

The spectral responsivity of the model is simulated by Matlab. Figure 4 shows the simulation result of this structure for a standard $0.5 \mu \mathrm{m}$ CMOS technology. As expected, there is an excellent UV responsivity and UV selectivity, as UV photons are absorbed near the surface of the structure and all the generated carriers are absorbed by the device, while the photo-generated carriers of longer wavelength are recombined at the short-circuited deeper junction. The maximal photocurrent is $1.38 \times 10^{-7} \mathrm{~A}$ at the wavelength of $460 \mathrm{~nm}$, corresponding a responsivity of $0.42 \mathrm{~A} / \mathrm{W}$; the photocurrent at longer wavelength is weak, it meets the properties of UV photodiode mainly well.

\section{Silicon Test Result}

The UV photodiode device under test is fabricated in a standard $0.5 \mu \mathrm{m}$ CMOS process, the microphotograph is shown in Figure 5. It concludes stripe-shaped anode, and short-circuited substrate electrode and cathode electrode. Two pads are connected with anode and cathode, respectively.

The responsivity is tested using the configuration as shown in Figure 6. The anode of the stripe-shaped UV photodiode is connected to a high precision amperemeter (with the accuracy of $1 \mathrm{fA}$ ), the cathode is connected with power source $\left(\mathrm{V}_{\mathrm{DD}}\right)$. The high precision amperemeter is connected with a resistance of $200 \mathrm{~K} \Omega$.

Placing the UV photodiode in a dark environment, external power supplies $\mathrm{V}_{\mathrm{DD}}=18 \mathrm{~V}$, incident optical wavelength is $400 \mathrm{~nm}, 460 \mathrm{~nm}, 520 \mathrm{~nm}, 585 \mathrm{~nm}, 605 \mathrm{~nm}$ and $630 \mathrm{~nm}$, respectively.

The measured curve of photo-current vs wavelength is shown in Figure 7. It has relatively high photo-current in UV spectral range; the maximal photo-current is $1.34 \times$ $10^{-7} \mathrm{~A}$ at the wavelength of $460 \mathrm{~nm}$. If translate photo-current into responsivity, that is to say, the maximal responsivity is $0.42 \mathrm{~A} / \mathrm{W}$ at the wavelength of $460 \mathrm{~nm}$. Comparing with simulation results, it's easy to see that silicon test results are agree with simulation results at the spectral range of UV.

\section{Conclusion}

In this work, a novel responsivity model is established

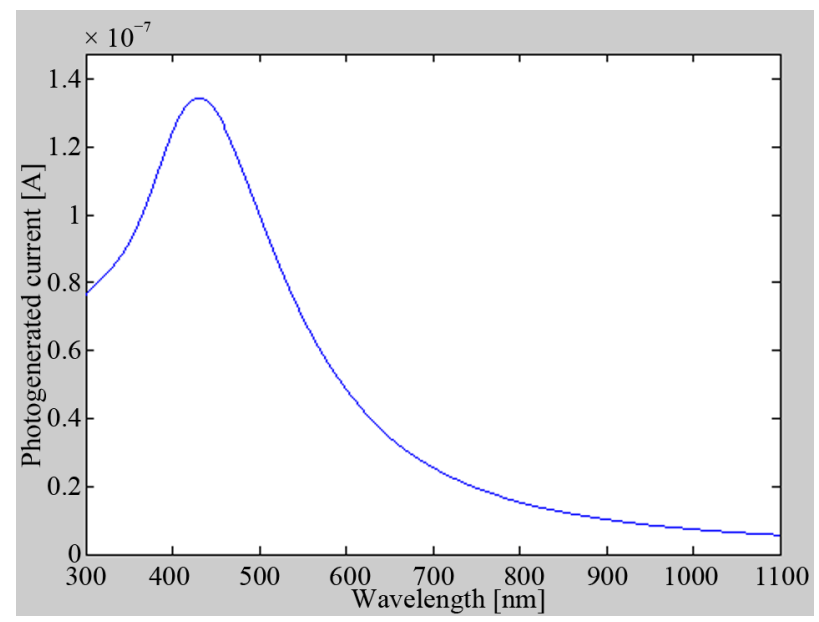

Figure 4. Simulation result of the responsivity model using Matlab.

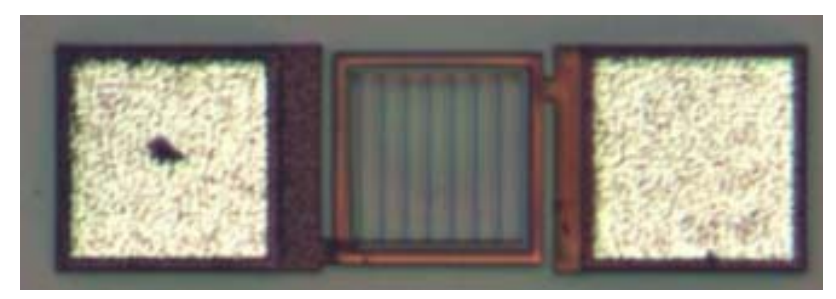

Figure 5. Microphotograph of stripe-shaped UV photodiode fabricated in a standard $0.5 \mu \mathrm{m}$ CMOS process. 


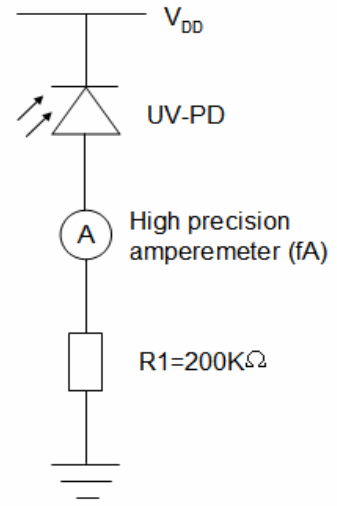

Figure 6. Configuration of test circuit for UV photodiode.

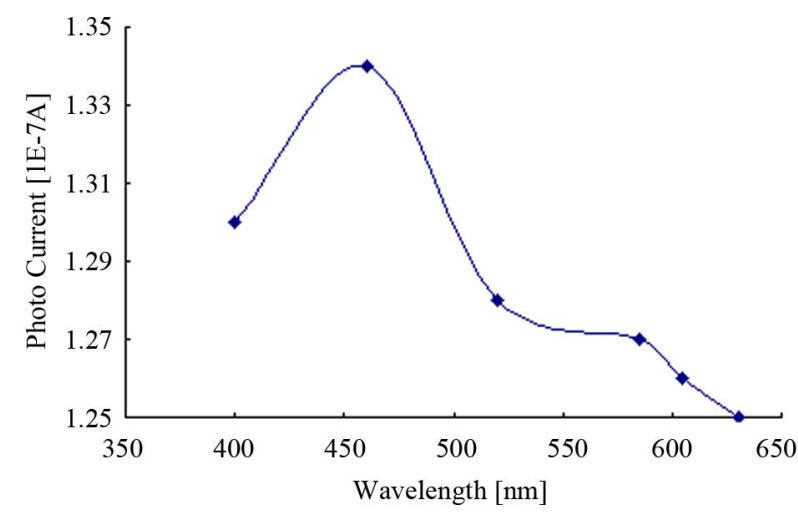

Figure 7. Measured photo-current vs wavelength.

for stripe-shaped UV photodiode. The structure is tapedout by $0.5 \mu \mathrm{m}$ CMOS process. Simulation results and silicon test results are both have maximal UV responsivity at UV spectral range. The mainly agreement between them demonstrate that this work can be used to analysis the optical properties of stripe-shaped UV photodiode.

\section{Acknowledgements}

This paper is supported by Scientific Research Fund of Hunan Provincial Education Department (11A116), by Key Project of Chinese Ministry of Education of China (212125) and by Hunan Provincial Natural Science Foundation of China (11JJ2036).

\section{REFERENCES}

[1] E. Charbon, "Towards Large Scale CMOS Single-Photon Detector Arrays for Lab-on-Chip Applications," Journal of Physics D: Applied Physics, Vol. 41, No. 9, 2008, Article ID: 094010. doi:10.1088/0022-3727/41/9/094010

[2] Z. Djuric, K. Radulovic, N. Trbojevic and Z. Lazic, "Silicon Resonant Cavity Enhanced UV Flame Detector," International Conference on Microelectronics, Vol. 1, 2002, pp. 239-242.

[3] M. P. Ulmer, "A Review of UV Detectors for Astrophysics: Past, Present, and Future," Proceedings of SPIE, Vol. 7222, 2009, Article ID: 722210. doi:10.1117/12.810039

[4] G. K. Li, P. Feng and N. J. Wu, "A Novel Monolithic Ultraviolet Image Sensor Based on a Standard CMOS Process," Journal of Semiconductors, Vol. 32, No. 10, 2011, Article ID: 105008.

[5] A. Ghazi, H. Zimmermann and P. Seegebrecht, "CMOS Photodiode with Enhanced Responsivity for the UV/Blue Spectral Range," IEEE Transactions on Electron Devices, Vol. 49, No. 7, 2002, pp. 1124-1128. doi:10.1109/TED.2002.1013266

[6] B. A. Saleh, M. M. Hayat and M. C. Teich, "Effect of Dead Space on the Excess Noise Factor and Time Response of Avalanche Photodiodes," IEEE Transactions on Electron Devices, Vol. 37, No. 9, 1990, pp. 19761984. doi:10.1109/16.57159

[7] M. M. Hayat, W. L. Sargeant and B. E. A. Saleh, "Effect of Dead Space on Gain and Noise in Si and GaAs Avalanche Photodiodes," IEEE Journal of Quantum Electronics, Vol. 28, No. 5, 1992. pp. 1360-1365. doi: $10.1109 / 3.135278$

[8] M. M. Hayat, B. E. A. Saleh and M. C. Teich, "Effect of Dead Space on Gain and Noise of Double-Carrier-Multiplication Avalanche Photodiodes," IEEE Transactions on Electron Devices, Vol. 39, No. 3, 1992, pp. 546-552. doi:10.1109/16.123476

[9] A. Pauchard, P.-A. Besse and R. S. Popovic, "A Silicon Blue/UV Selective Stripe-Shaped Photodiode," Sensors and Actuators A: Physical, Vol. 76, No. 1-3, 1999, pp. 172-177. doi:10.1016/S0924-4247(99)00006-0

[10] A. Pauchard, P.-A. Besse and R. S. Popovic, "Dead Space Effect on the Wavelength Dependence of Gain and Noise in Avalanche Photodiodes," IEEE Transactions on Electron Devices, Vol. 47, No. 9, 2000, pp. 1685-1693. doi:10.1109/16.861578

[11] A. Alexandre, F. Dadouche and P. Garda, "Two Dimensional Model for Lateral Photodiode," International Conference on Design and Test of Integrated Systems in Nanoscale, Tunis, 5-7 September 2006, pp. 294-298.

[12] I. R. Rawlings, "Optical Absorption in Silicon Monoxide," Journal of Physics D: Applied Physics, Vol. 1, No. 6, 1733, p. 733. 